\title{
Tensile Strength and Pseudo-elasticity of YAG Laser Spot Melted Ti-Ni Shape Memory Alloy Wires
}

\author{
Yasuhito Ogata*, Masaya Takatugu, Takeshi Kunimasa, Keisuke Uenishi and Kojiro F. Kobayashi \\ Department of Manufacturing Science, Graduate School of Engineering, Osaka University, Suita 565-0871, Japan
}

In order to investigate the applicability of laser micro welding to the fabrication of medical devices, Ti-Ni shape memory alloy wires with pseudo-elasticity were micro spot melted by using YAG laser. By changing the melting parameters, such as laser power or pulse duration, the evolution in microstructure, tensile strength and pseudo-elasticity was investigated. Melted metal width decreased with decreasing the input energy and pulse duration. The microstructure of melted areas was cellular dendrite structures and that of heat affected areas was grown cell structure with a cell size of about $3 \sim 10 \mu \mathrm{m}$. Tensile strength of spot melted wires was $30 \%$ lower than that of base metal, and the fracture occurred in melted areas with a brittle fracture surface. Although melted areas and heat affected areas did not show a pseudo-elasticity due to the larger grain size, minimizing the width of melted metal improved the overall pseudo-elasticity of spot melted samples. For the sample wires that were prepared by spot melting of cold rolled wires and by the subsequent heat treatment for shape memorizing, the pseudo-elasticity was improved because heat affected zone recrystallized to form fine grains.

(Received November 25, 2003; Accepted January 23, 2004)

Keywords: titanium-nickel shape memory alloy, YAG laser, laser welding, tensile strength, pseudo-elasticity, biomaterial

\section{Introduction}

Ti-Ni shape memory alloy wires were widely used for medical devices, such as stent, catheter guide wire, or coil anchor, owing to their superior machinability, corrosion resistance in a biological environment and unique pseudoelasticity. These micro medical devices have played an important role in the recent progress on medical treatments. ${ }^{1-4)}$ The recent demands against these medical devices lie on the development of finer and more complicated devices. For instance, stent and coil anchor, which are implanted into blood vessel, were required to be applied for finer or more complicated parts of human body. Although laser cutting or hand made technique has been applied to fabricate them at the present moment, but it takes high costs and it is difficult to fabricate finer or more complicated medical devices.5) Consequently, establishment of micro laser welding technology is considered to be one of the available methods for the fabrication of the micro medical devices. The authors have reported the fair laser spot weldability of the stainless steel wires, which is widely prevailing as a biomaterial. ${ }^{5-7}$ ) The spot melted SUS304 wires exhibited almost the same tensile strength and corrosion resistance as base metal.

In this research, in order to investigate the applicability of laser micro welding to the fabrication of medical devices, Ti$\mathrm{Ni}$ shape memory alloy wires were micro spot melted by YAG laser. By the measurements of tensile strength and pseudo-elasticity as well as by microstructural observation of the joints, the effects of laser conditions such as laser power or pulse duration on the joint reliability were discussed. Moreover, the effect of shape memory heat treatment after micro spot melting was discussed on the improvement of tensile strength or pseudo-elasticity.

\section{Experimental Procedure}

Ti-Ni based shape memory alloy wires (NT-E4 wires) with a diameter of $0.15 \mathrm{~mm}$ or $0.35 \mathrm{~mm}$ were used in this study. In this research, two types of NT-E4 wires were prepared. One is wire already shape memory heat treated and the other is as cold rolled wire. In this paper, as cold rolled wires and already shape memorized wires will be abbreviated to be CR and SM wires, respectively. The chemical composition and transformation temperature of NT-E4 wires are shown in Table 1 and Table 2. These wires were spot melted by a single pulse laser irradiation using Nd-YAG laser equipment (Lumonic JK701) in an Ar shielding atmosphere. Laser frequency, focal distance and beam radius were $20 \mathrm{~Hz}$, $80 \mathrm{~mm}$ and $400 \mu \mathrm{m}$, respectively. Laser power was varied from 0.2 to $4.0 \mathrm{~J}$, and pulse duration was changed to be 2,5 , 10 , and $20 \mathrm{~ms}$. The schematic illustration of laser melting process is shown in Fig. 1.

Microstructural observation of spot melted and heat affected area was performed by using optical microscope,

Table 1 Chemical composition of NT-E4 wire.

\begin{tabular}{ccc}
\hline & \multicolumn{2}{c}{ Chemical composition (mass\%) } \\
\hline \multirow{2}{*}{ NT-L } & $\mathrm{Ti}$ & $\mathrm{Ni}$ \\
\cline { 2 - 3 } & 44.09 & 55.91 \\
\hline
\end{tabular}

Table 2 Transformation temperature of NT-E4 wire.

\begin{tabular}{ccc}
\hline NT-L & \multicolumn{2}{c}{ Transformation temperature $(\mathrm{K})$} \\
\cline { 2 - 3 } diameter $(\mathrm{mm})$ & $A_{\mathrm{f}}$ & $M_{\mathrm{s}}$ \\
\hline 0.15 & 287.9 & 261.7 \\
0.35 & 286.8 & 261.5 \\
\hline
\end{tabular}

\section{YAG laser}

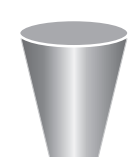

Wire specimen

Fig. 1 The schematic illustrations of laser melting process. 
scanning electron microscope (SEM) and transmission electron microscope (TEM). Tensile strength tests were conducted at a cross-head speed of $3.3 \times 10^{-3} \mathrm{~mm} / \mathrm{s}$. Pseudo-elastic properties were estimated by the measurement of residual strain after loading and off-loading the stress of $4 \%$ or $8 \%$. Single spot melted NT-E4 wires with a gage length of $100 \mathrm{~mm}$ and a grab length of $150 \mathrm{~mm}$ were applied to tensile strength and pseudo-elasticity tests. Heat treatment condition to shape memorize of the spot melted CR wires was holding the samples at $803 \mathrm{~K}$ for $120 \mathrm{~s}$ in an Ar atmosphere followed by quenching in iced water.

\section{Results and Discussion}

\subsection{Morphology of spot melted SM wires}

The typical cross section of spot melted SM wire is shown in Fig. 2. Any melting imperfections like cracks or porosities were not observed. However, when laser power were not sufficient, the morphology of melted metals became irregular. With increasing the laser power, penetration depth and melted area were increased, but too much laser power caused the break down of wires. Melted metal width decreased with decreasing the input energy or pulse duration.

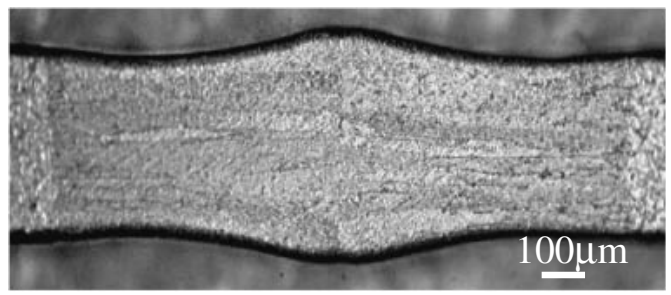

Fig. 2 The typical microstructure of spot melted SM wire.

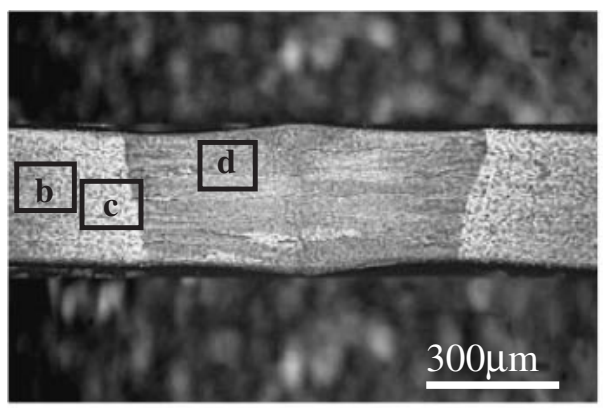

(a) General view

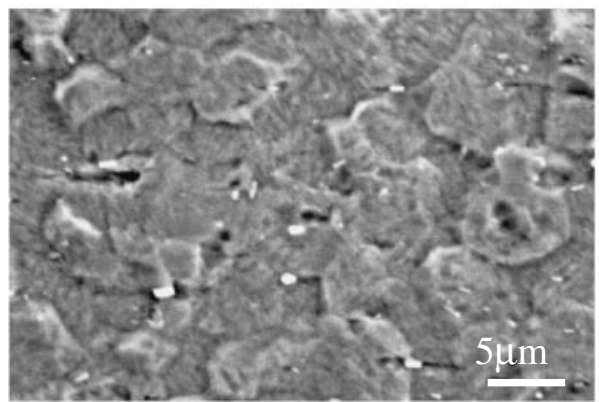

(c) Heat affected area

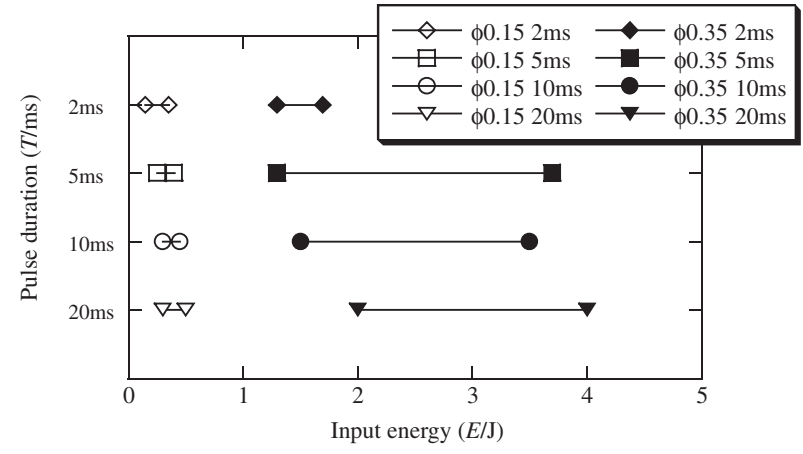

Fig. 3 Laser condition range where reasonable melting can be achieved for SM wires.

Laser condition range where fully penetrated reasonable melting can be achieved was shown in Fig. 3. The reasonable melting of $0.35 \mathrm{~mm}$ diameter wires can be achieved by the heat input from 1.5 to $3.5 \mathrm{~J}$ at a pulse duration of $10 \mathrm{~ms}$. These heat input value are slightly smaller than those for spot melting of SUS304 wires. On the contrary, the condition range for NT-E4 became smaller than that for SUS304. Especially for the melting with shorter pulse duration or melting of the thinner $(\phi 0.15 \mathrm{~mm})$ wires, the condition range became much smaller. In conclusion, reasonable spot melting of SM wires can be achieved by the smaller heat input, but it needs more precise control of laser conditions compared with that of SUS304 wires.

\subsection{Microstructure of spot melted SM wires}

The typical microstructures of spot melted SM wire observed with SEM or with TEM are shown in Fig. 4 and Fig. 5.

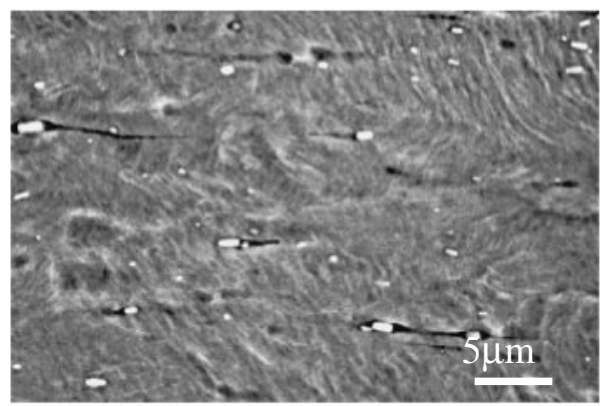

(b) Base metal

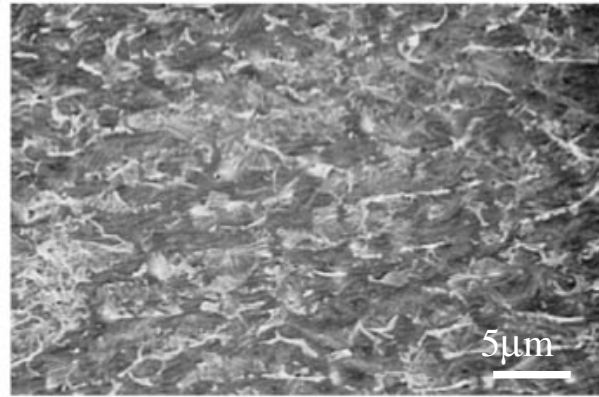

(d) Melted metal

Fig. 4 SE images of spot melted SM wires. 


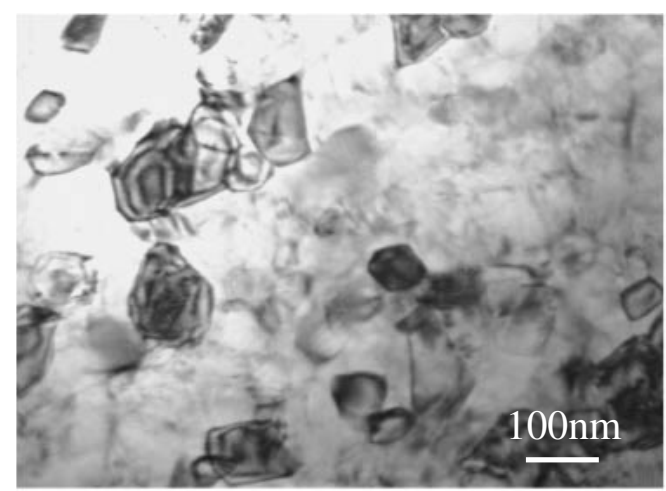

(a) Base metal

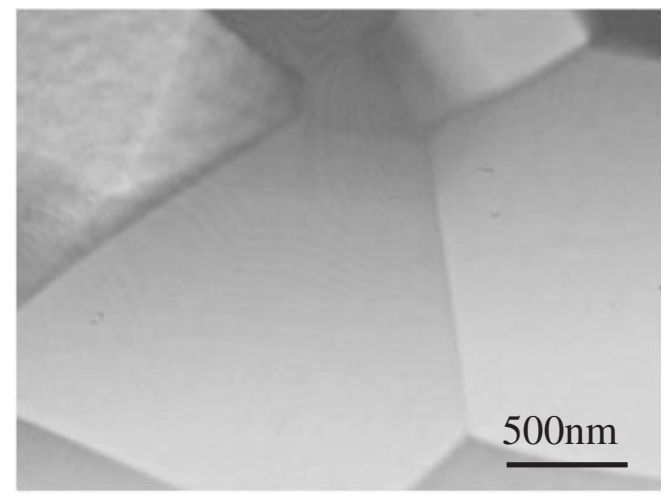

(b) Heat affected area

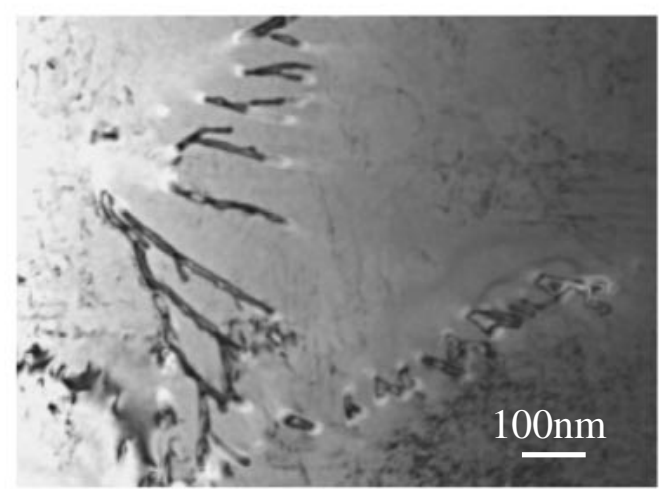

(c) Melted metal

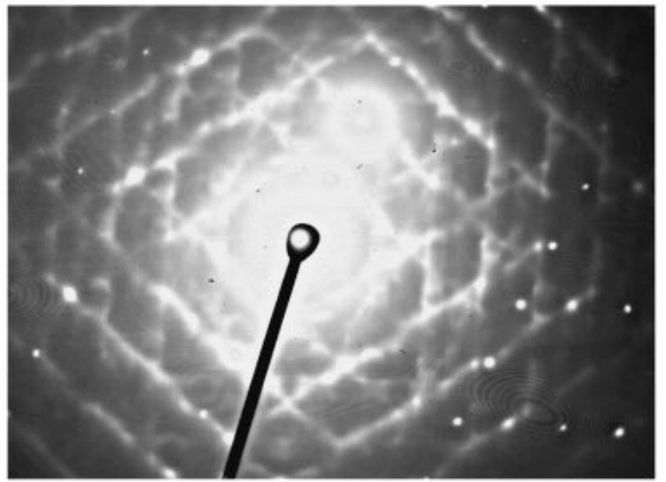

(d) Diffraction pattern of melted metal

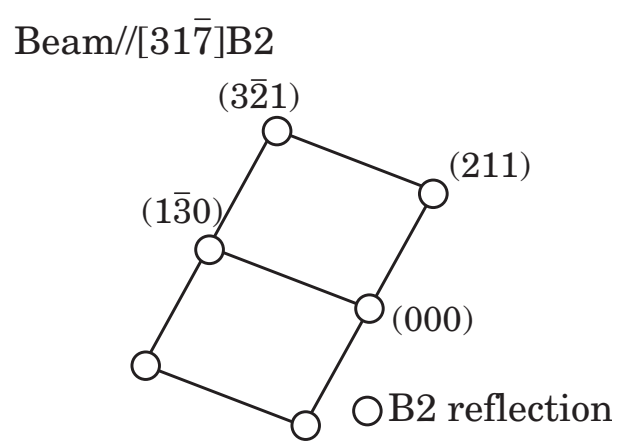

(e) Key diagram of melted metal

Fig. 5 TEM micrographs and diffraction patterns of spot melted SM wires.

The base metal exhibited about $0.1 \mu \mathrm{m}$ fine equiaxed recrystallized microstructure, which is introduced by the deformation during cold rolling and the subsequent heat treatment for shape memorizing. It was confirmed by electron diffraction analysis that the base metal was composed of B2 Ti-Ni phase.

The melted metal exhibited a dendrite microstructure that grew from fusion boundary to center of the melted metal. The grain size of melted metal was about $1 \sim 3 \mu \mathrm{m}$ due to the rapid solidification effect. It was confirmed by the electron diffraction analysis that the melted metal still retained B2 structure.

In the heat affected area, fine equiaxed grains of base material were coarsened to the size of $3 \sim 10 \mu \mathrm{m}$ by heat affection during laser irradiation.

\subsection{Tensile strength of spot melted SM wires}

The effect of laser condition on tensile strength for spot melted SM wires is shown in Fig. 6. Although the tensile strength changed a bit with a laser power or a pulse duration, tensile strength of the single spot melted SM wires was about $900 \mathrm{MPa}$, which is about $70 \%$ of that of SM wires. Figure 7 shows the typical fracture surface and the fracture path of spot melted SM wires after tensile strength test. While base metal exhibited a ductile fracture surface after tensile strength test, all of the spot melted samples fractured brittle in the melted areas. This degradation in tensile strength is considered to be due to the absence of pseudo-elasticity in 


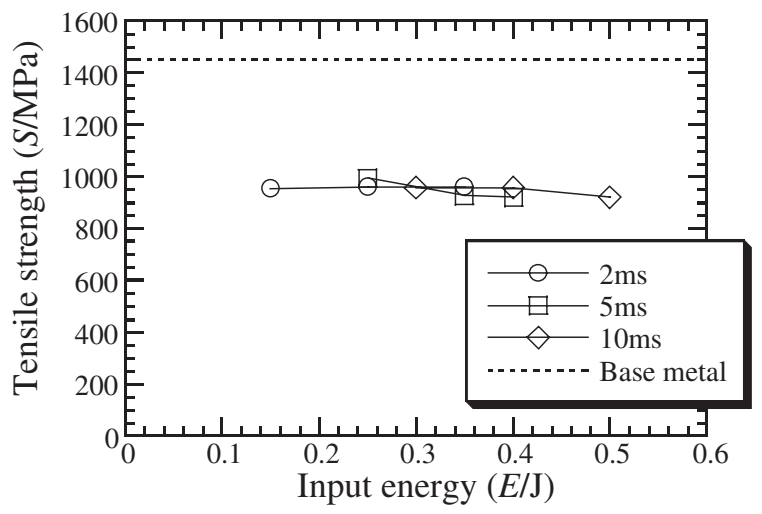

(a) Diameter $0.15 \mathrm{~mm}$

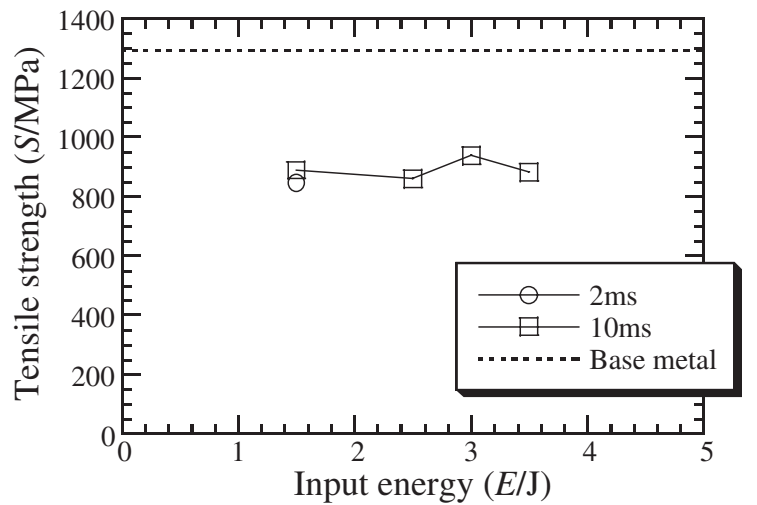

(b) Diameter $0.35 \mathrm{~mm}$

Fig. 6 The effect of heat input and pulse duration on tensile strength for spot melted SM wires.
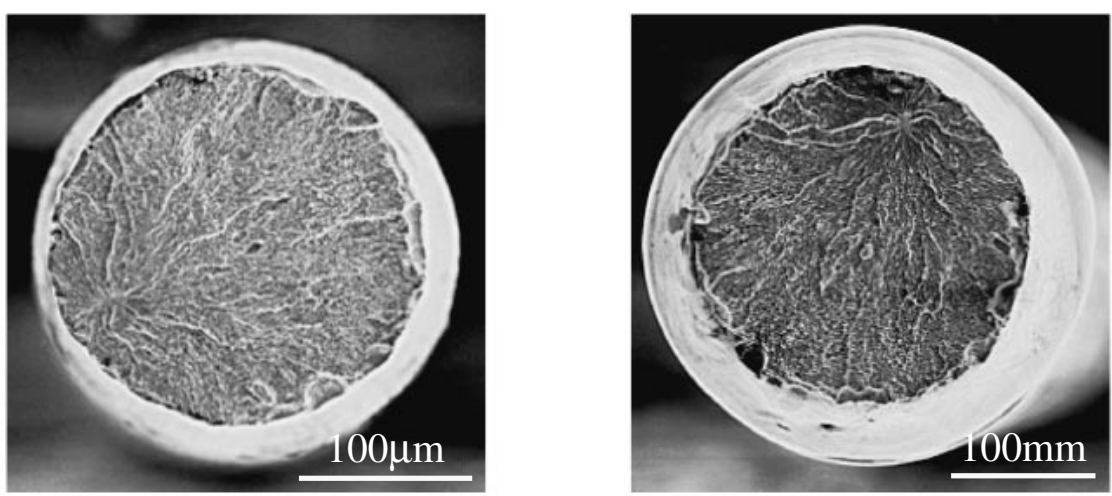

(a) Fracture surfaces
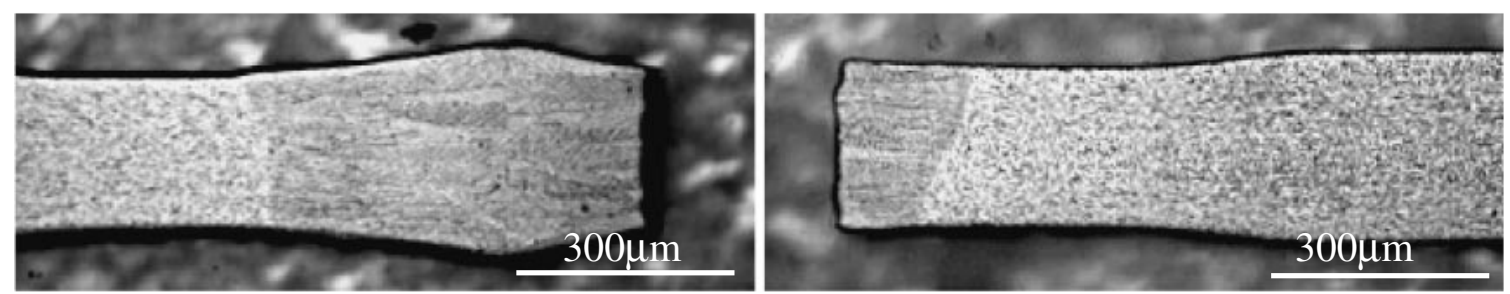

(b) Fracture paths

Fig. 7 Fracture surfaces and fracture paths of spot melted SM wires after tensile test.

melted region where the stress concentrates.

\subsection{Pseudo-elasticity}

Pseudo-elastic behaviors of as received SM wire and single spot melted SM wire are shown in Fig. 8. These stress-strain curves were obtained during loading and unloading the $4 \%$ or $8 \%$ of stress. While as received wires showed the stress-strain curve typical to the pseudo-elasticity where any residual strain cannot be detected, the strain was not released completely even by unloading the strain for spot melted wires. Residual strain after loading and unloading the strain of $4 \%$ or $8 \%$ were $0.23 \%$ or $0.58 \%$, respectively. Table 3 shows the transformation temperature of spot melted region for SM wires. $A_{\mathrm{f}}$ and $M_{\mathrm{S}}$ temperature measured by DSC were almost same as those of base metal although the transformation peaks became broader. It can be concluded that spot melted region exhibits the $\mathrm{B} 2 \rightarrow M_{\mathrm{S}}$ transformation, but it becomes less sensitive. For the development of pseudoelasticity, it is essential to minimize the grain size to $1 \mu \mathrm{m}$ so that any movement of dislocations or slips does not occur within the grains. Because of the fine microstructure of the base metal, it is enough to exercise the good pseudoelasticity. Though spot melted wires retain B2 structure, this degradation in pseudo-elasticity lies on the fact that heat affected area and melted area did not show pseudo-elasticity due to the larger grain size than $1 \mu \mathrm{m}$.

The relation between width of melted area and residual strain for spot melted SM wires were shown in Fig. 9. The 


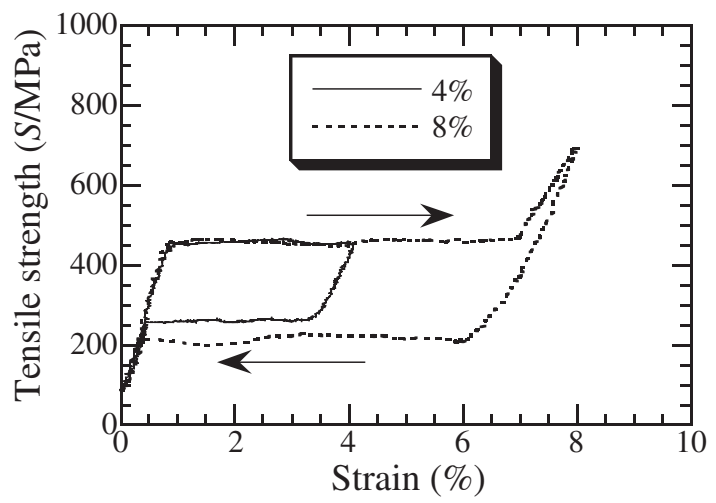

(a) SM wire

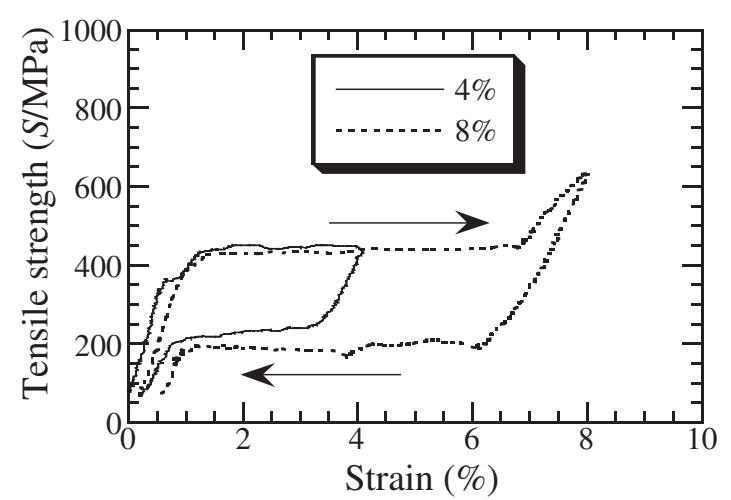

(b) Single spot melted SM wire

Fig. 8 Pseudo-elasticity behaviors of SM wires.

Table 3 Transformation temperatures of spot melted SM wires.

\begin{tabular}{ccc}
\hline & \multicolumn{2}{c}{ Transformation temperature $(\mathrm{K})$} \\
\cline { 2 - 3 } & $A_{\mathrm{f}}$ & $M_{\mathrm{s}}$ \\
\hline Weld metal & 299.1 & 258.3 \\
Base metal & 286.8 & 261.5 \\
\hline
\end{tabular}

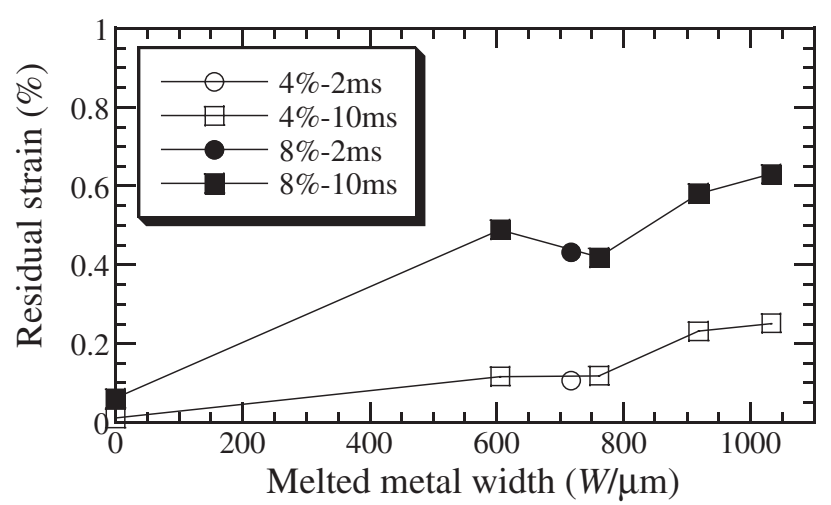

Fig. 9 The relation between width of melted area and residual strain.

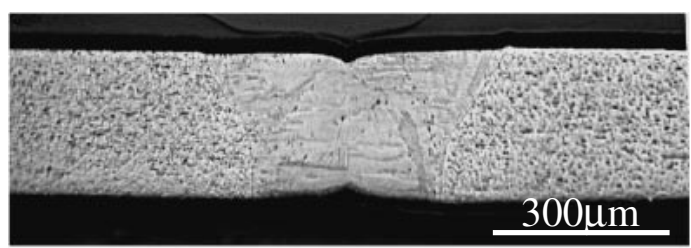

(a) $2 \mathrm{~ms}, 1.3 \mathrm{~J}$

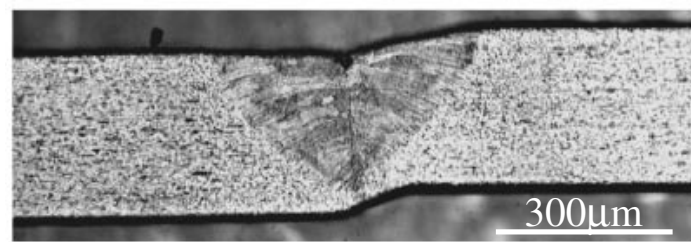

(c) $10 \mathrm{~ms}, 1.5 \mathrm{~J}$ residual strain decreased with decreasing the melted area width. Consequently, the width of melted area should be minimized by the optimization of laser conditions such as using a small pulse duration. In the present research, since the width of melted area was enough small compared with the length of test wires, the spot melted SM wires totally exhibited pseudo-elastic behavior comparable with SM wires.

\subsection{Spot melting of as cold rolled wires followed by shape memory heat treatment}

\subsubsection{Morphology of spot melted CR wires}

The typical cross sections of spot melted CR wires are shown in Fig. 10. Any melting imperfections like cracks and porosities were not observed. For the spot melting of $0.35 \mathrm{~mm}$ diameter CR wires in a pulse duration of $10 \mathrm{~ms}$, laser conditions range where reasonable melting can be achieved were almost same as that for the SM wires. The spot melted CR wires without shape memory property were likely to be misaligned by thermal shock especially for a shorter pulse duration. Consequently, it was essential to more precisely control the laser condition for the spot melting of $\mathrm{CR}$ wires than that of SM wires.

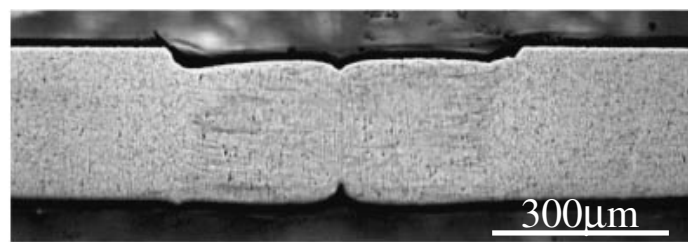

(b) $2 \mathrm{~ms}, 1.5 \mathrm{~J}$

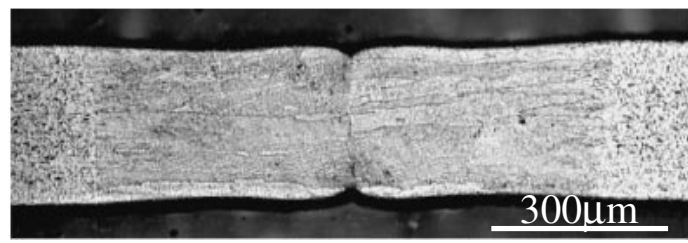

(d) $10 \mathrm{~ms}, 3.5 \mathrm{~J}$

Fig. 10 The typical cross sections of spot melted CR wires. 


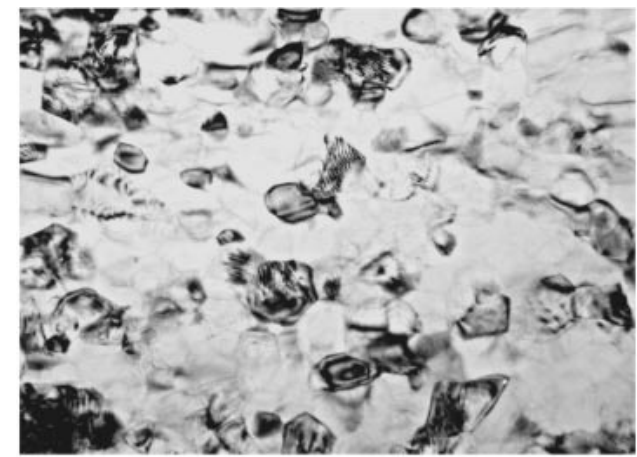

$100 \mathrm{~nm}$

(a) Base metal

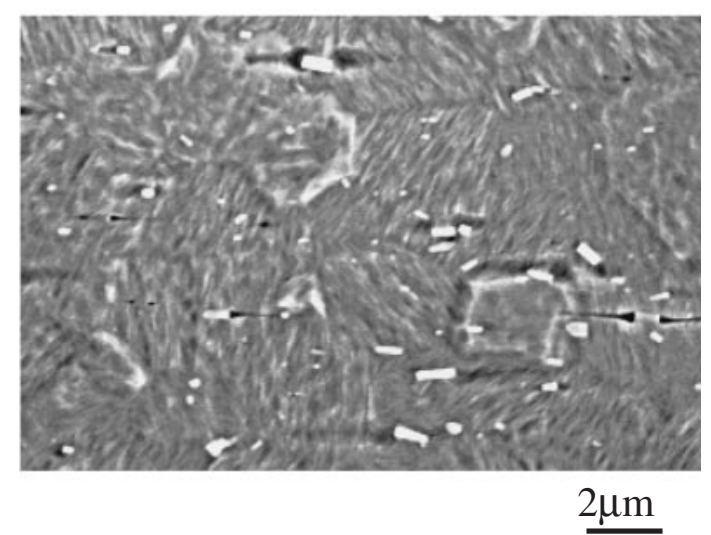

(b) Heat affected area

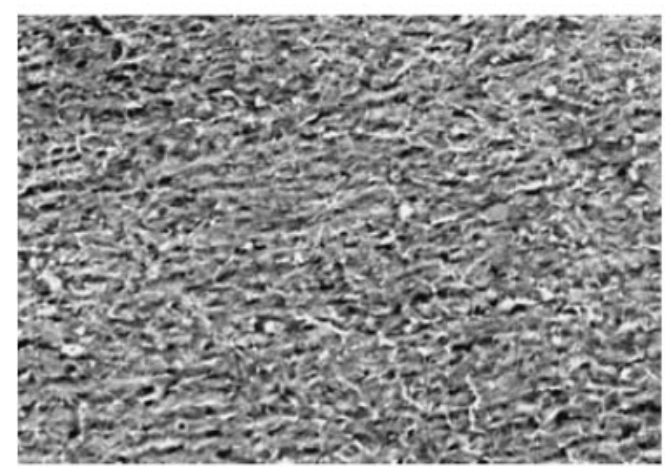

(c) Melted metal $\underline{5 \mu \mathrm{m}}$

Fig. 11 The typical microstructures of CR wires after spot melting and shape memorizing.

\subsubsection{Microstructure of $\mathrm{CR}$ wires after spot melting and shape memorizing}

Figure 11 shows the typical microstructures of CR wires after spot melting and shape memorizing. The base metal and melted metal exhibited almost the same microstructures as SM wires. In the heat affected area, many fine equiaxed grains whose size were about $1 \mu \mathrm{m}$ were formed by the recrystallization, although fine equiaxed grains were grown to the size of $3 \sim 10 \mu \mathrm{m}$ for the spot melted SM wires. Both heat affected and melted area still retained B2 structure.

\subsubsection{Tensile strength of CR wires after spot melting} and shape memorizing

The effect of laser conditions on tensile tests of CR wires after spot melting and shape memorizing is shown in Fig. 12.

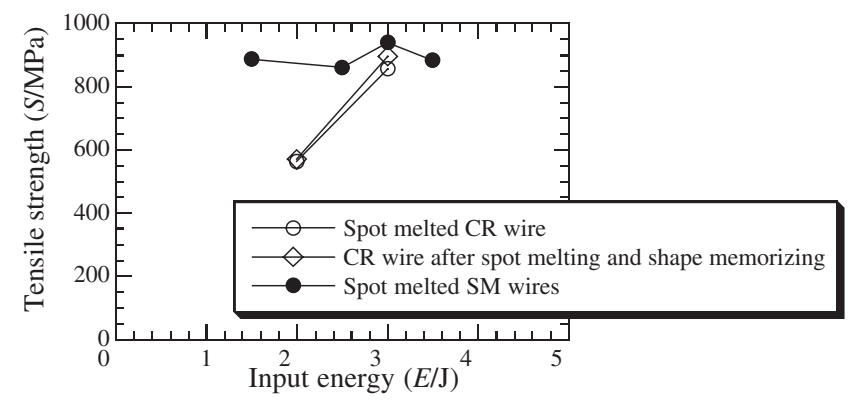

Fig. 12 The effect of laser conditions on tensile tests of spot melted CR wires.

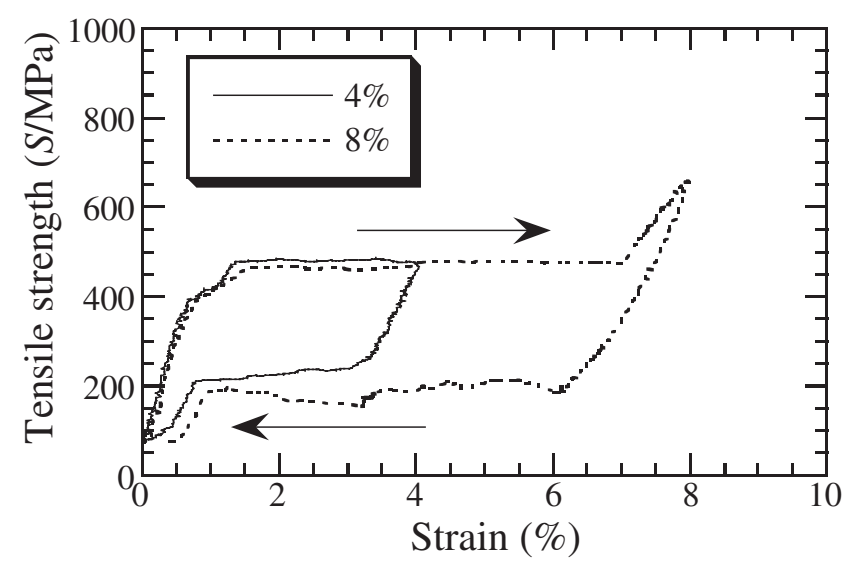

Fig. 13 Pseudo-elasticity behaviors of CR wires after spot melting and shape memorizing.

Tensile strength of $\mathrm{CR}$ wires after spot melting and shape memorizing were about $900 \mathrm{MPa}$, which is almost same as spot melted SM wires. However, it should be noted that tensile strength decreased even by minimizing laser power, although spot melted SM wires did not shown these decrease. These decrease of tensile strength were caused by the misalignment of CR wires after melting shown in Fig. 10. In conclusion, fair laser condition range to obtain samples with comparable tensile strength became smaller for the CR wires than for the SM wires.

\subsubsection{Pseudo-elasticity of CR wires after spot melting and shape memorizing}

Pseudo-elastic behaviors of CR wires after spot melting and shape memorizing are shown in Fig. 13. Although the strain was not recovered completely like spot melted SM wires, the residual strain after loading and unloading the strain of $4 \%$ or $8 \%$ loaded strain were $0.106 \%$ or $0.44 \%$, respectively. Since these values were smaller than those of spot melted SM wires, it was confirmed that the technique of spot melting and the subsequent shape memorizing the CR wires is effective to obtain NT-E4 joints with sound pseudoelasticity.

\section{Conclusion}

In this research, in order to investigate the applicability of laser welding to the fabrication of medical devices, microstructure, tensile strength and pseudo-elasticity were inves- 
tigated for the laser spot melted NT-E4 shape memory wires. The following results were obtained in this study:

(1) Any melting imperfections like cracks and porosities were not observed. Reasonable spot melting of SM wires can be achieved by the smaller heat input, but it needs more precise control of laser conditions compared with that of SUS304 wires.

(2) The microstructure of melted areas were cellular dendrite structures whose grain size was about $1 \sim 3 \mu \mathrm{m}$ and that of heat affected areas were coarsened cells with the cell size of about $3 \sim 10 \mu \mathrm{m}$.

(3) Tensile strength of spot melted SM wires was about $900 \mathrm{MPa}$ that was 30 percent lower than that of base metal. Fracture occurred in melted areas and the fracture surface revealed a brittle structure.

(4) The width of melted area could be minimized to $650 \mu \mathrm{m}$ by optimizing the laser conditions so that the spot melted SM wires totally exhibited pseudo-elastic behavior comparable with base materials.

(5) The technique of spot melting and the subsequent shape memorizing the CR wires is effective to obtain NT-E4 joints with sound pseudo-elasticity, although it needs more precise control of laser condition.

\section{Acknowledgements}

The authors thank the stuff in Laserx Co., Ltd. and Furukawa Electronic Industry Co., Ltd. for the experimental help about laser irradiation and for supplying the NT-E4 base materials, respectively. This research is financially supported by JSPS grant-in aid for Scientific Research.

\section{REFERENCES}

1) T. Tuboi: The Review of Laser Engineering 28, No. 7 (2000) 413-424.

2) T. Yoneyama and E. Kobayashi: Journal of JSNDI 49, No. 7, 420-424.

3) S. Miyazaki: Materia Japan 35 (1996) 179.

4) H. Hamanaka and T. Tuthiya: Ferrum 27 (1997) 30-35.

5) A. Hirose, M. Uchihara, T. Araki, K. Araki and M. Kondo: J. Japan Inst. Metals 54 (1990) 262-269.

6) K. Uenishi, M. Takatugu, M. Seki, T. Kunimasa, K. F. Kobayashi, T. Ikeda and A. Tuboi: Mater. Trans. 43 (2002) 3083-3087.

7) K. Uenishi, M. Seki, T. Kunimasa, M. Takatsugu, K. F. Kobayashi, T. Ikeda and A. Tuboi: Proceedings of Third International Symposium on Laser Precision Microfabrication, 2002 Osaka Japan, SPIE Vol. 4830 (2003) 57-62. 